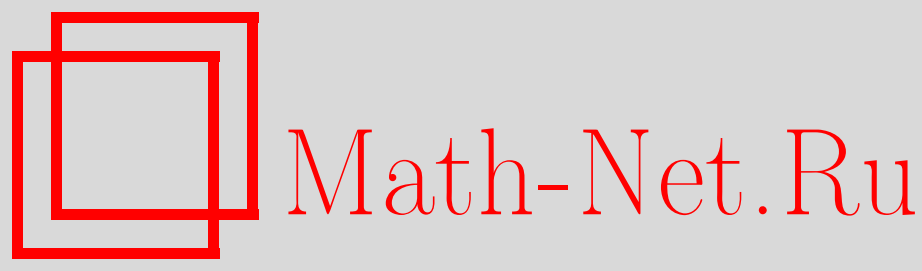

В. В. Борзов, Е. В. Дамаскинский, Обобщенные когерентные состояния для осцилляторов, связанных с qполиномами Шарлье, ТМФ, 2008, том 155, номер 1, 3946

DOI: https://doi.org/10.4213/tmf6191

Использование Общероссийского математического портала Math-Net.Ru подразумевает, что вы прочитали и согласны с пользовательским соглашением http://www . mathnet.ru/rus/agreement

Параметры загрузки:

IP: 35.174 .16 .151

26 апреля 2023 г., 08:45:02

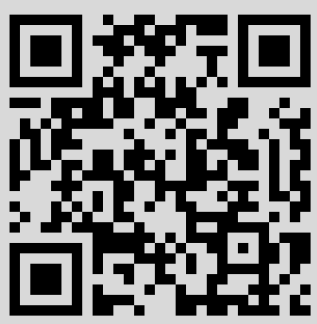




\section{ОБОБЩЕННЫЕ КОГЕРЕНТНЫЕ СОСТОЯНИЯ ДЛЯ ОСЦИЛЛЯТОРОВ, СВЯЗАННЫХ С $q$-ПОЛИНОМАМИ ШАРЛЬЕ}

Продолжено исследование обобщенных когерентных состояний для осцилляторо-подобных систем, связанных с заданным семейством ортогональных многочленов. Рассмотрен случай обобщенных осцилляторов, порождаемых $q$-полиномами Шарлье.

Ключевые слова: деформированные осцилляторы, когерентные состояния, ортогональные многочлены, $q$-полиномы Шарлье.

\section{1. ВВЕДЕНИЕ}

Роль, которую играют когерентные состояния в квантовой теории, трудно переоценить. Основные сведения, относящиеся к теории когерентных состояний, могут быть найдены в работах [1], [2]. Обширная библиография, включающая работы до 2000 г., имеется в обзоре [3]. В последнее время возрос интерес к построению и исследованию когерентных состояний для осцилляторо-подобных систем, связанных с семействами ортогональных многочленов (см., например, [4]-[7]).

В предыдущих работах авторов (см., например, [8], [9] и цитированные там работы авторов) был предложен новый метод построения осцилляторо-подобных систем, связанных с семействами ортогональных многочленов подобно тому, как обычный бозонный осциллятор связан с полиномами Эрмита, и развита схема построения обобщенных когерентных состояний для таких систем (см. подробное изложение в [9]). В настоящей работе мы применим эту конструкцию к случаю $q$-полиномов Шарлье.

\section{2. ПОСТРОЕНИЕ ОБОБЩЕННОГО ОСЦИЛЛЯТОРА}

Обобщенный осциллятор определяется перестановочными соотношениями:

$$
\left[a_{\mu}^{-}, a_{\mu}^{+}\right]=2(B(\mathcal{N}+I)-B(\mathcal{N})), \quad\left[\mathcal{N}, a_{\mu}^{ \pm}\right]= \pm a_{\mu}^{ \pm}
$$

* Санкт-Петербургский государственный университет телекоммуникаций им. М. А. Бонч-Бруевича, Санкт-Петербург, Россия. E-mail: vadim@VB6384.spb.edu

${ }^{\dagger}$ Санкт-Петербургский военный инженерно-технический университет, Санкт-Петербург, Россия. E-mail: evd@pdmi.ras.ru 
(см. обозначения в [9]). Отметим, что если $B(\mathcal{N})$ является многочленом по $\mathcal{N}$ (это верно в случае классических полиномов как непрерывного, так и дискретного аргумента), то алгебру $\mathcal{A}_{\mu}$, порождаемую операторами $a_{\mu}^{ \pm}, \mathcal{N}$ с перестановочными соотношениями (1), будем называть алгеброй обобщенного осциллятора, связанного с канонической системой полиномов $\left\{\psi_{n}(x)\right\}_{n=0}^{\infty}$, ортонормированных относительно вероятностной меры $\mu$. Ниже символ $\mathcal{A}_{\mu}$ мы будем использовать также и для обозначения самого осциллятора. Центр алгебры $\mathcal{A}_{\mu}$ порождается элементом

$$
\mathcal{C}=2 B(\mathcal{N})-a_{\mu}^{+} a_{\mu}^{-} .
$$

Если же $B(\mathcal{N})$ не является многочленом, но удовлетворяет рекуррентному соотношению

$$
\alpha B(\mathcal{N}+I)+B(\mathcal{N})+\gamma B(\mathcal{N}-I)=0, \quad \alpha \neq 0, \quad \gamma \neq 0
$$

(что справедливо в случае $q$-аналогов классических полиномов), то, введя вместо $\mathcal{N}$ два новых генератора $B(\mathcal{N}+I)$ и $D(\mathcal{N})=B(\mathcal{N}+I)-B(\mathcal{N})$, мы получим алгебру с перестановочными соотношениями

$$
\begin{gathered}
{\left[a_{\mu}^{-}, a_{\mu}^{+}\right]=2 D(\mathcal{N}), \quad\left[B(\mathcal{N}+I), a_{\mu}^{-}\right]=-a_{\mu}^{-} D(\mathcal{N}), \quad[B(\mathcal{N}+1), D(\mathcal{N})]=0} \\
{\left[B(\mathcal{N}+I), a_{\mu}^{+}\right]=a_{\mu}^{+}\left(\frac{\gamma}{\alpha} D(\mathcal{N})-\frac{1+\alpha+\gamma}{\alpha} B(\mathcal{N}+I)\right)} \\
{\left[D(\mathcal{N}), a_{\mu}^{-}\right]=a_{\mu}^{-}\left(\frac{1+\alpha+\gamma}{\gamma} B(\mathcal{N}+I)-\frac{1+2 \gamma}{\gamma} D(\mathcal{N})\right)} \\
{\left[D(\mathcal{N}), a_{\mu}^{+}\right]=a_{\mu}^{+}\left(\frac{\gamma-\alpha}{\alpha} D(\mathcal{N})-\frac{1+\alpha+\gamma}{\alpha} B(\mathcal{N}+I)\right) .}
\end{gathered}
$$

Алгебру обобщенного осциллятора, порождаемую этими соотношениями, мы будем обозначать тем же символом $\mathcal{A}_{\mu}$. Центр этой алгебры порождается элементом

$$
\mathcal{C}_{1}=2 B(\mathcal{N}+I)-a_{\mu}^{-} a_{\mu}^{+} .
$$

\section{3. q-ОСЦИЛЛЯТОР ШАРЛЬЕ}

3.1. $q$-Полиномы Шарлье. $q$-Полиномы Шарлье (связанные с полиномами Аль-Салама-Карлица второго рода) $C_{n}^{\mu}(x \mid q), 0<q<1,0<\mu<1$, определяются формулой [10]

$$
C_{n}^{\mu}(x \mid q)={ }_{2} \phi_{0}\left(\begin{array}{c|c}
q^{-n}, x \\
-
\end{array} \mid q, \frac{1}{\mu} q^{1+n}\right), \quad x=q^{-s},
$$

где ${ }_{2} \phi_{0}-$ базисная гипергеометрическая функция,

$$
{ }_{2} \phi_{0}\left(\begin{array}{c|c}
a, b \\
-
\end{array} \mid q, z\right)=\sum_{k=0}^{\infty}(-1)^{k} q^{-\left(\begin{array}{c}
k \\
2
\end{array}\right)} \frac{(a, b ; q)_{k}}{(q ; q) k} z^{k} .
$$

Здесь используются стандартные обозначения

$$
(a ; q)_{n}=\prod_{k=0}^{n-1}\left(1-a q^{k}\right), \quad(a ; q)_{\infty}=\prod_{k=0}^{\infty}\left(1-a q^{k}\right), \quad(a, b ; q)_{n}=(a ; q)_{n}(b ; q)_{n}
$$


Рекуррентные соотношения для полиномов $C_{n}^{\mu}(x \mid q)$ имеют вид

$$
\begin{aligned}
\mu q^{-n-1} C_{n+1}^{\mu}(x \mid q)+\left(q^{-n}-1\right) C_{n-1}^{\mu}(x \mid q) & =\left((\mu+q) q^{-n-1}-x\right) C_{n}^{\mu}(x \mid q), \quad n>0, \\
C_{0}^{\mu}(x \mid q) & =1 .
\end{aligned}
$$

Эти полиномы ортогональны,

$$
\sum_{s=0}^{\infty} C_{m}^{\mu}\left(q^{-s} \mid q\right) C_{n}^{\mu}\left(q^{-s} \mid q\right) \rho(s)=\frac{(q ; q)_{n}}{\mu^{n}} \delta_{m n},
$$

относительно дискретной положительной меры

$$
\rho(s)=(\mu ; q)_{\infty} \frac{\mu^{s} q^{s(s-1)}}{(q, \mu ; q)_{s}}, \quad 0<\mu, q<1 .
$$

Следуя [11], введем перенормированные полиномы $\psi_{n}(x, \mu)$, положив

$$
C_{n}^{\mu}(x \mid q)=\gamma_{n} \psi_{n}(x, \mu), \quad \gamma_{n}=(q ; q)_{n}^{1 / 2} \mu^{-n / 2}, \quad n \geqslant 0,
$$

рекуррентные соотношения для которых при $n \geqslant 0$ имеют вид

$$
\begin{gathered}
x \psi_{n}(x, \mu)=b_{n} \psi_{n+1}(x, \mu)+a_{n} \psi_{n}(x, \mu)+b_{n-1} \psi_{n-1}(x, \mu), \\
\psi_{0}(x, \mu)=1,
\end{gathered}
$$

где

$$
a_{n}=(\mu+q) q^{-n-1}, \quad b_{n}=-q^{-n-1} \sqrt{\mu\left(1-q^{n+1}\right)}, \quad n \geqslant 0 .
$$

Условие ортогональности для полиномов запишется в виде

$$
\sum_{s=0}^{\infty} \psi_{m}\left(q^{-s}, \mu\right) \psi_{n}\left(q^{-s}, \mu\right) \rho(s)=\delta_{m n}
$$

Используя $q$-интеграл Джексона

$$
\int_{x}^{\infty} f(t) d_{q} t:=x(1-q) \sum_{1}^{\infty} q^{-n} f\left(q^{-n} x\right),
$$

условие ортогональности (10) можно переписать в виде

$$
\psi_{m}(1, \mu) \psi_{n}(1, \mu)+\int_{1}^{\infty} \psi_{m}(t, \mu) \psi_{n}(t, \mu) \tilde{\rho}(t) d_{q} t=\delta_{m n}
$$

где $\tilde{\rho}(t)=\rho\left(-\log _{q} t\right)$.

Построенное семейство многочленов образует ортонормированный базис в гильбертовом пространстве $\mathcal{H}_{\nu}=L^{2}(\mathbb{R} ; d \nu)$ относительно вероятностной меры $d \nu(x)$. Поскольку

$$
\sum_{n=0}^{\infty} b_{n}^{-1}<\infty
$$

то проблема моментов для матрицы Якоби, связанной с рекуррентными соотношениями $(9)$, является неопределенной и мера $d \nu(x)$ определена неоднозначно. 
3.2. q-Осциллятор Шарлье. В гильбертовом пространстве $\mathcal{H}_{\nu}$, рассматриваемом как реализация пространства Фока, определим оператор координаты $X_{\nu}$, задав его действие на элементы базиса $\{|n\rangle\}_{n=0}^{\infty}$ (такого, что $\langle x \mid n\rangle=\psi_{n}(x, \mu)$ ) формулами

$$
X_{\nu}|n\rangle=b_{n}|n+1\rangle+a_{n}|n\rangle+b_{n-1}|n-1\rangle \text {, }
$$

в соответствии с рекуррентными соотношениями (9). Известно, что линейный оператор $X_{\nu}$, определенный с помощью (14) на линейной оболочке элементов базиса, симметричен и имеет индексы дефекта $(1,1)$. Следовательно, он имеет бесконечное семейство самосопряженных расширений $X_{\nu}^{\theta}$, индексируемых параметром $\theta \in[0, \pi]$. Фиксируя самосопряженное расширение $X_{\nu}^{\theta}$, можно построить $N$-экстремальную меру $d \nu_{\theta}$ (см. [12]).

Приведем соответствующие результаты [12], выбрав для определенности значение $\theta=0$. В этом случае носитель $\Pi_{0}=\left\{x_{k}\right\}_{k=-\infty}^{\infty}$ меры $d \nu_{0}$ состоит из точек, которые являются корнями трансцендентного уравнения

$$
\psi_{0}(x, \mu)+x \sum_{k=0}^{\infty}(-1)^{k} \sqrt{\frac{\{2 k-2\} ! !}{\{2 k-1\} ! !}} \psi_{2 k-1}(x, \mu)=0,
$$

где $\{s\}=b_{s-1}^{2} / b_{0}^{2}, s \geqslant 1,\{0\}=0$. Нагрузки $\sigma_{0}\left(x_{k}\right)$ в точках носителя $\Pi_{0}$ меры $d \nu_{0}$ равны

$$
\sigma_{0}\left(x_{k}\right)=-x_{k} \frac{Q_{1}\left(x_{k}\right)+\sum_{l=2}^{\infty}(-1)^{l-1} \sqrt{\frac{\{2 l-2\} ! !}{\{2 l-1\} ! !}} Q_{2 l-1}\left(x_{k}\right)}{\left.\left[\frac{d}{d x}\left(P_{0}(x)+\sum_{l=1}^{\infty}(-1)^{l} \sqrt{\frac{\{2 l-2\} ! !}{\{2 l-1\} ! !}} x P_{2 l-1}(x)\right)\right]\right|_{x_{k}}} .
$$

Здесь многочлены $P_{n}=\psi_{n}(x, \mu)$ и $Q_{n}(x, \mu)$ - полиномы 1-го и 2-го рода для матрицы Якоби, связанной с оператором $X_{\nu}$. Для симметричной матрицы Якоби их явные выражения приведены в [12]. Далее, следуя [11], введем оператор импульса $P_{\nu}$, задав его действие на элементах базиса в $\mathcal{H}_{\nu}$ соотношениями

$$
P_{\nu}|n\rangle=i\left(b_{n}|n+1\rangle-b_{n-1}|n-1\rangle\right)+a_{n}|n\rangle .
$$

Этот оператор, так же как и $X_{\nu}$, симметричен в $\mathcal{H}_{\nu}$, имеет индексы дефекта $(1,1)$ и бесконечное семейство самосопряженных расширений. Мы будем рассматривать то самосопряженное расширение оператора $P_{\nu}$, которое согласовано с выбранным выше самосопряженным расширением оператора $X_{\nu}$ так, чтобы соответствующие операторы рождения и уничтожения были взаимно сопряженными. Сохраним за этими расширениями обозначения $X_{\nu}$ и $P_{\nu}$. Следуя методу, описанному в [13], оператор $P_{\nu}$ можно реализовать в пространстве $\mathcal{H}_{\nu}$ как некоторый дифференциально-разностный оператор. Ниже мы получим его явный вид из сравнения наших результатов с результатами работ [14], [15].

Далее, следуя [11], определим осцилляторо-подобную систему, которую будем называть $q$-осциллятором Шарлье. Введем операторы обобщенной координаты $\widetilde{X}_{\nu}$ и обобщенного импульса $\widetilde{P}_{\nu}$, положив

$$
\widetilde{X}_{\nu}=\operatorname{Re}\left(X_{\nu}+P_{\nu}\right), \quad \widetilde{P}_{\nu}=-i \operatorname{Im}\left(X_{\nu}-P_{\nu}\right),
$$


а также операторы рождения и уничтожения

$$
\tilde{a}_{\nu}^{+}=\frac{1}{\sqrt{2}}\left(\widetilde{X}_{\nu}+i \widetilde{P}_{\nu}\right), \quad \tilde{a}_{\nu}^{-}=\frac{1}{\sqrt{2}}\left(\widetilde{X}_{\nu}-i \widetilde{P}_{\nu}\right),
$$

действующие на базис пространства Фока $\mathcal{H}_{\nu}$ согласно формулам

$$
\tilde{a}_{\nu}^{+}|n\rangle=\sqrt{2} b_{n}|n+1\rangle, \quad \tilde{a}_{\nu}^{-}|n\rangle=\sqrt{2} b_{n-1}|n-1\rangle .
$$

Гамильтониан q-осциллятора Шарлье выберем в виде

$$
H=\widetilde{X}_{\nu}^{2}+\widetilde{P}_{\nu}^{2}
$$

Из результатов работы [11] следует, что спектр этого гамильтониана имеет вид

$$
H|n\rangle=\lambda_{n}|n\rangle,
$$

где

$$
\begin{gathered}
\lambda_{0}=2 b_{0}^{2}=2 \mu(1-q) q^{-2}, \\
\lambda_{n}=2\left(b_{n-1}^{2}+b_{n}^{2}\right)=2 \mu(1-q) q^{-2(n+1)}\left([n+1]_{q}-q^{2}[n]_{q}\right) ;
\end{gathered}
$$

здесь $[n]_{q}=\left(1-q^{n}\right) /(1-q)$ - обычное $q$-число.

Так как

$$
2 b_{n-1}^{2}-2 b_{n}^{2}=2 \mu \frac{1-q}{q^{2 n+1}}\left([n+1]_{q}-q^{2}[n]_{q}\right),
$$

то перестановочные соотношения для лестничных операторов (19) имеют вид

$$
\left[\tilde{a}_{\nu}^{-}, \tilde{a}_{\nu}^{+}\right]=2 \mu(1-q) q^{-2(N+1)}\left([N+1]_{q}-q^{2}[N]_{q}\right),
$$

где $N$ - оператор, нумерующий элементы базиса: $N|n\rangle=n|n\rangle$.

3.3. Связь с результатами работ Аски-Суслова. В работах [14], [15] pacсматривалась дискретная реализация q-осциллятора Арика-Куна, операторы рождения и уничтожения которого удовлетворяют перестановочным соотношениям

$$
a_{q}^{-} a_{q}^{+}-q a_{q}^{+} a_{q}^{-}=\mathbf{1}
$$

Рассматривалась реализация этих операторов в пространстве $\mathcal{H}_{\mathrm{AS}}=L^{2}\left(\mathbb{R}_{+} ; d \omega\right)$, где мера $d \omega$ имеет носитель в точках $s=0,1, \ldots$ с нагрузкой $\sigma(s)=1$. Функции Шарлье

$$
\varphi_{n}(s ; \mu)=\sqrt{\rho(s)} \psi_{n}\left(q^{-s}, \mu\right)
$$

образуют в пространстве $\mathcal{H}_{\mathrm{AS}}$ ортонормированный базис:

$$
\sum_{s=0}^{\infty} \varphi_{m}(s ; \mu) \varphi_{n}(s ; \mu)=\delta_{m n} .
$$

Операторы $a_{q}^{-}$и $a_{q}^{+}$в этом пространстве имеют вид

$$
\begin{aligned}
& a_{q}^{-}=\frac{1}{\sqrt{1-q}}\left(\sqrt{\mu} q^{s}-\sqrt{\left(1-q^{s+1}\right)\left(1-\mu q^{s}\right)} e^{\partial_{s}}\right), \\
& a_{q}^{+}=\frac{1}{\sqrt{1-q}}\left(\sqrt{\mu} q^{s}-e^{-\partial_{s}} \sqrt{\left(1-q^{s+1}\right)\left(1-\mu q^{s}\right)}\right),
\end{aligned}
$$


где $\partial_{s}=d / d s, e^{\alpha \partial_{s}} f(s)=f(s+\alpha)$. Эти операторы действуют на векторах базиса в $\mathcal{H}_{\mathrm{AS}}$ согласно формулам

$$
a_{q}^{-} \varphi_{n}(s ; \mu)=\sqrt{[n]_{q}} \varphi_{n-1}(s ; \mu), \quad a_{q}^{+} \varphi_{n}(s ; \mu)=\sqrt{[n+1]_{q}} \varphi_{n+1}(s ; \mu) .
$$

Если построить унитарное преобразование, связывающее пространства $\mathcal{H}_{\nu}$ и $\mathcal{H}_{\mathrm{AS}}$, то из формул (28) получаем явные выражения для операторов рождения и уничтожения в нашем варианте $q$-осциллятора Шарлье

$$
\begin{aligned}
& \tilde{a}_{\nu}^{+}=-\sqrt{2 \mu(1-q)} \rho^{-1 / 2} a_{q}^{+} \rho^{1 / 2} \rho^{-(N+I)}, \\
& \tilde{a}_{\nu}^{-}=-\sqrt{2 \mu(1-q)} \rho^{-1 / 2} a_{q}^{-} \rho^{1 / 2} \rho^{-N} .
\end{aligned}
$$

\section{4. КОГЕРЕНТНЫЕ СОСТОЯНИЯ ДЛЯ $q$-ОСЦИЛЛЯТОРА ШАРЛЬЕ}

Определим когерентные состояния типа Барута-Жирарделло для $q$-осциллятора Шарлье стандартным образом [16], [17]:

$$
\tilde{a}_{\nu}^{-}|z\rangle=z|z\rangle, \quad|z\rangle=\mathcal{N}^{-1 / 2}\left(|z|^{2}\right) \sum_{n=0}^{\infty} \frac{z^{n}}{\left(\sqrt{2} b_{n-1}\right) !}|n\rangle,
$$

где $\left(\sqrt{2} b_{n-1}\right) !=\left(\sqrt{2} b_{0}\right)\left(\sqrt{2} b_{1}\right) \ldots\left(\sqrt{2} b_{n-1}\right)$. Учитывая соотношение

$$
\left(2 b_{n-1}^{2}\right) !=(2 \mu(1-q))^{n} q^{-n(n+1)}[n]_{q} !=\left(\frac{2 \mu}{q}\right)^{n} q^{-n^{2}}(q ; q)_{n},
$$

вычисляем нормирующий множитель

$$
\mathcal{N}\left(|z|^{2}\right)=\sum_{n=0}^{\infty}\left(\frac{q|z|^{2}}{2 \mu}\right)^{n} \frac{q^{n^{2}}}{(q ; q)_{n}}={ }_{0} \phi_{1}\left(\begin{array}{c}
- \\
0
\end{array} q ; \frac{q^{2}|z|^{2}}{2 \mu}\right)
$$

и перекрытие двух когерентных состояний

$$
\left\langle z_{1} \mid z_{2}\right\rangle=\sum_{n=0}^{\infty}\left(\frac{q \bar{z}_{1} z_{2}}{2 \mu}\right)^{n} \frac{q^{n^{2}}}{(q ; q)_{n}}={ }_{0} \phi_{1}\left(\begin{array}{c}
- \\
0
\end{array} \mid q ; \frac{q^{2} \bar{z}_{1} z_{2}}{2 \mu}\right) .
$$

Далее имеем

$$
\begin{aligned}
\langle x \mid z\rangle & =\left({ }_{0} \phi_{1}\left(\begin{array}{c}
- \\
0
\end{array} \mid q ; \frac{q^{2}|z|^{2}}{2 \mu}\right)\right)^{-1 / 2} \sum_{n=0}^{\infty}\left(\frac{z^{n}}{(2 \mu / q)^{n / 2}}\right)^{n} \frac{q^{n^{2} / 2}}{\sqrt{(q ; q)_{n}}} \psi_{n}(x, \mu)= \\
& =\left({ }_{0} \phi_{1}\left(\overline{-} \mid q ; \frac{q^{2}|z|^{2}}{2 \mu}\right)\right)^{-1 / 2} \sum_{n=0}^{\infty} \frac{q^{\left(\begin{array}{c}
n \\
2
\end{array}\right)}}{(q ; q)_{n}}\left(\frac{q z}{\sqrt{2}}\right)^{n} C_{n}^{\mu}(x \mid q) .
\end{aligned}
$$

Используя связь $q$-полиномов Шарлье с полиномами Аль-Салама-Карлица 2-го рода [10]

$$
C_{n}^{\mu}(x \mid q)=V_{n}^{\mu / q}(x ; q) q^{\left(\begin{array}{c}
n \\
2
\end{array}\right)}\left(-\frac{q}{\mu}\right)^{n}
$$


где

$$
V_{n}^{a}(x ; q)=(-a)^{n} q^{-\left(\begin{array}{c}
n \\
2
\end{array}\right)}{ }_{2} \phi_{0}\left(\begin{array}{c|c}
q^{-n}, x & q ; \frac{q^{n}}{a} \\
- &
\end{array}\right),
$$

и производящую функцию для полиномов $V_{n}^{a}$

$$
\sum_{n=0}^{\infty} \frac{q^{2\left(\begin{array}{c}
n \\
2
\end{array}\right)}}{(q ; q)_{n}} V_{n}^{a}(x ; q) t^{n}=(a t ; q)_{\infty} \cdot{ }_{1} \phi_{1}\left(\begin{array}{c}
x \\
a t
\end{array} \mid q ; t\right),
$$

соотношение (35) можно переписать в виде [10]

$$
\begin{aligned}
\langle x \mid z\rangle & =\left({ }_{0} \phi_{1}\left(\begin{array}{c}
- \\
0
\end{array} \mid q ; \frac{|z|^{2}}{2 \mu}\right)\right)^{-1 / 2} \sum_{n=0}^{\infty} \frac{q^{2\left(\begin{array}{c}
n \\
2
\end{array}\right)}}{(q ; q)_{n}}\left(-\frac{q^{2} z}{\sqrt{2} \mu}\right)^{n} V_{n}^{\mu / q}(x ; q)= \\
& =\left({ }_{0} \phi_{1}\left(\begin{array}{c}
- \\
0
\end{array} \mid q ; \frac{|z|^{2}}{2 \mu}\right)\right)^{-1 / 2}\left(-\frac{q z}{\sqrt{2}} ; q\right)_{\infty}{ }_{1} \phi_{1}\left(\begin{array}{c}
x \\
-\frac{q z}{\sqrt{2}}
\end{array} \mid q ;-\frac{q^{2} z}{\sqrt{2} \mu}\right) .
\end{aligned}
$$

Осталось доказать соотношение (сверх)полноты

$$
\iint_{\mathbb{C}} \widehat{W}\left(|z|^{2}\right)|z\rangle\langle z| d^{2} z=\mathbf{1}
$$

т.е. построить меру

$$
d \mu\left(|z|^{2}\right)=\widehat{W}\left(|z|^{2}\right) d^{2} z, \quad d^{2} z=d(\operatorname{Re} z) d(\operatorname{Im} z) .
$$

Известно [16], что для этого надо решить классическую проблему моментов Стилтьеса

$$
\pi \int_{0}^{\infty} x^{n} W(x) d x=\left(\frac{2 \mu}{q}\right)^{n} q^{-n^{2}}(q ; q)_{n}
$$

где

$$
W(x)=\frac{\widehat{W}(x)}{\mathcal{N}(x)}, \quad x=|z|^{2} .
$$

При замене переменных

$$
x=\frac{2 \mu}{q} y, \quad \frac{2 \pi \mu}{q} W\left(\frac{2 \mu}{q} y\right)=\widetilde{W}(y),
$$

проблема моментов принимает вид

$$
\int_{0}^{\infty} y^{n} \widetilde{W}(y) d y=q^{-n^{2}}(q ; q)_{n} .
$$

В работе [18] было получено решение этой задачи:

$$
\widetilde{W}(y)=\frac{1-q}{q^{2}} \sum_{k=0}^{\infty} y \cdot{ }_{2} \phi_{0}\left(\begin{array}{c}
0,0 \\
-
\end{array} \mid q ; q^{-2} y\right)\left(\delta\left(y-q^{-(k-1)}\right)+\delta\left(y-q^{k+2}\right)\right),
$$

так что

$$
\begin{gathered}
\widehat{W}(x)=\frac{1-q}{2 \pi \mu q}{ }_{0} \phi_{1}\left(\begin{array}{c}
- \\
0
\end{array} \mid q ; q^{-2} \frac{x}{2 \mu}\right) \sum_{k=0}^{\infty} q \frac{x}{2 \mu}{ }_{2} \phi_{0}\left(\begin{array}{c}
0,0 \\
-
\end{array} \mid q ; q^{-1} \frac{x}{2 \mu}\right) \times \\
\times\left(\delta\left(q \frac{x}{2 \mu}-q^{-(k-1)}\right)+\delta\left(q \frac{x}{2 \mu}-q^{k+2}\right)\right) .
\end{gathered}
$$


В результате для меры $d \mu\left(|z|^{2}\right)$ имеем

$$
\begin{aligned}
& d \mu\left(|z|^{2}\right)=\frac{1-q}{4 \pi \mu^{2}}{ }_{0} \phi_{1}\left(\begin{array}{c}
- \\
0
\end{array} \mid q ; q^{2} \frac{|z|^{2}}{2 \mu}\right) \times \\
& \quad \times \sum_{k=0}^{\infty}|z|^{2}{ }_{2} \phi_{0}\left(\begin{array}{c}
0,0 \\
-
\end{array} \mid q ; q \frac{|z|^{2}}{2 q \mu}\right)\left(\delta\left(q \frac{|z|^{2}}{2 \mu}-q^{-(k-1)}\right)+\delta\left(q \frac{|z|^{2}}{2 \mu}-q^{k+2}\right)\right) d^{2} z .
\end{aligned}
$$

Отметим в заключение, что, как было показано в [17], на когерентных состояниях соотношение неопределенности переходит в равенство.

Благодарности. Авторы признательны оргкомитету конференции "Классические и квантовые интегрируемые системы" за представленную им возможность участия в столь представительном совещании. Авторы благодарны П.П. Кулишу за внимание к работе и полезные замечания. Данное исследование осуществлялось при поддержке РФФИ (грант № 06-01-00451).

\section{Список литературы}

[1] А. М. Переломов, Обобщенные когерентные состояния и их применения, Наука, М., 1987.

[2] Дж. Клаудер, Э. Сударшан, Основы квантовой оптики, Мир, М., 1979.

[3] V. V. Dodonov, J. Opt. B, 4:1 (2002), R1-R33.

[4] R. Floreanini, L. Vinet, Lett. Math. Phys., 22:1 (1991), 45-54.

[5] Е. В. Дамаскинский, П. П. Кулиш, Записки науч. сем. ЛОМИ, 199 (1992), 81-90.

[6] N. M. Atakishiyev, E. I. Jafarov, Sh. M. Nagiyev, K. B. Wolf, Rev. Mexicana Fis., 44:3 (1998), 235-244; arXiv: math-ph/9807035.

[7] A. Odzijewicz, M. Horowski, A. Tereszkiewicz, J. Phys. A, 34 (2001), 4353-4376.

[8] В. В. Борзов, Е. В. Дамаскинский, Записки науч. сем. ПОМИ, 335 (2006), 75-99.

[9] В. В. Борзов, ТМФ, 153:3 (2007), 363-380.

[10] R. Koekoek, R.F. Swarttouw, The Askey sheme of hypergeometric orthogonal polynomials and its q-analogue, Report № 94-05, Delft University of Technology, 1994; arXiv: math. CA/9602214.

[11] V.V. Borzov, Integral Transforms Spec. Funct., 12:2 (2001), 115-138; arXiv: math.CA/0002226.

[12] V. V. Borzov, E. V. Damaskinsky, P. P. Kulish, Rev. Math. Phys., 12:5 (2000), 691-710.

[13] V. V. Borzov, E. V. Damaskinsky, Integral Transforms Spec. Funct., 13:6 (2002), 547-554; arXiv: math.QA/0101215.

[14] R. Askey, S. K. Suslov, J. Physics A, 26 (1993), L693-L698; arXiv: math/9307206.

[15] R. Askey, S. K. Suslov, Lett. Math. Phys., 29 (1993), 123-132; arXiv: math/9307207.

[16] J. R. Klauder, K. A. Penson, J.-M. Sixdeniers, Phys. Rev. A, 64:1 (2001), 013817.

[17] V. V. Borzov, E. V. Damaskinsky, "Coherent states and uncertainty relations for generalized oscillators connected with the given families of orthogonal polynomials", Proc. Intern. Semin. Day on DIFFRACTION 2005, ed. I. V. Andronov, St. Petersburg State Univ., St. Petersburg, 2005, 40-49.

[18] В. В. Борзов, Е. В. Дамаскинский, Записки науч. сем. ПОМИ, 308 (2004), 48-66. 